\title{
Temperature- and Time-Dependent Penetration of Surface Structures in Thermal Joining of Plastics to Metals
}

\author{
Klaus Schricker ${ }^{1, a^{*}}$ and Jean Pierre Bergmann ${ }^{1, b}$ \\ ${ }^{1}$ Technische Universität IImenau, Production Technology Group \\ aklaus.schricker@tu-ilmenau.de, binfo.fertigungstechnik@tu-ilmenau.de
}

\begin{abstract}
Keywords: laser, joining, hybrid, half-section setup, wetting, simulation, surface penetration
\end{abstract}
\begin{abstract}
The temperature- and time-dependent penetration of surface structures is examined in thermal joining between polypropylene and aluminum. Experimental and numerical investigations were carried out for spot joints in order to describe the main effects on structure penetration. Further investigations were performed in a half-section setup to gain information directly from the joining zone. The thermal expansion of the thermoplastic material as well as the temperature distribution in the melting layer were identified as key parameters for structure filling on the metal surface.
\end{abstract}

\section{Introduction and State of the Art}

The use of hybrid metal-plastic components gains importance in several fields of application, e. $g$. for lightweight design in automotive industry or functionalization in domestic appliance technology. Thereby, joining technology is a key parameter for realizing hybrid structures wherefore several processes are described within the state of the art, e. g. mechanical fasting, adhesive bonding or thermal joining. Thermal joining has advantages over competing processes due to the elimination of joining elements, filler materials, adhesives and long curing times.

Different thermal joining processes are known, e. g. induction joining, friction joining or laserbased joining. All processes have in common that a metal is in contact with a thermoplastic in overlap [1] or butt joint configuration [2]. The metal sheet is heated by the energy input during the process. The polymer is molten due to the heat transfer between both materials, penetrates the surface of the metal joining partner and thus wets it. After solidification, a solid joint is formed.

In the state of the art, several investigations were carried out on this process and especially the form fit between thermoplastic material and metal sheet was identified as key parameter to achieve a high joint strength. Therefore, a wide field of investigations was carried out on surface preparation of the metal sheet on surface morphology and topography [3], various structure sizes [4], different structuring processes [5] and the resulting tensile strength [4-6]. Besides surface characterization and optimization of the joint strength, the surface penetration and wetting were subject of investigations. For this, a sufficient wetting time of several seconds was mentioned in investigations of [7] in order to form well bonded metal-plastic joints. On the other hand, much shorter joining times were realized in thermal joining for other processes, e. g. below $1 \mathrm{~s}$ in resistance joining [8].

A theoretical approach of surface penetration is given in [9] for nanoscale surface structures in oxide layers by plasticized or molten plastic during the joining process. Rheology, capillary flow and pressure conditions in the structure were considered as key variables. However, relatively long joining times of several minutes were required for penetrating the nanometer-scaled structures and the temperature is considered indirectly, e.g. by viscosity. Further knowledge can be gained if joining temperatures and times are directly taken into account and generalized. A direct transfer to microscopic or macroscopic structures is basically conceivable, but completely different structural geometries, pressure conditions and physico-chemical interactions between metal and plastic exist. Therefore, this article focuses on the interaction between temperature distribution, different joining times and material properties on surface penetration in thermal joining. 


\section{Experimental Setup}

\section{Materials and Surface-Pretreatment}

Investigations were carried out on Polypropylene (PP) as non-polar material to exclude physicochemical interactions between plastic melt and the metal surface which could affect the surface penetration. A thickness of $5 \mathrm{~mm}$ was chosen for the thermoplastic materials to fulfill the requirements of the semi-infinite body in order to avoid heat accumulation and further effects on the temperature distribution. The thermoplastics were characterized by differential scanning calorimetry (Netzsch DSC $204 \mathrm{~F} 1$ Phoenix, heating rate: $10 \mathrm{~K} \cdot \mathrm{min}^{-1}$, according to $[10,11]$ ) to gain information about the melting interval ( $\mathrm{T}_{\mathrm{im}}$ : begin of melting interval, $\mathrm{T}_{\mathrm{em}}$ : end of melting interval). Thermogravimetric analysis (Netzsch TG $209 \mathrm{~F} 1 \mathrm{Iris}$, heating rate: $10 \mathrm{~K} \cdot \mathrm{min}^{-1}$, nitrogen atmosphere, according to [12]) was carried out to determine the initial decomposition temperature $\left(\mathrm{T}_{\mathrm{d}}\right)$ at which bubble formation occurs (see also [13]). The aluminum alloy EN AW 6082 with a thickness of $1.5 \mathrm{~mm}$ was used as metal joining partner. A pretreatment of the metal surface was realized by a pulsed fiber laser (RofinPowerLine F20, average power: $20 \mathrm{~W}$, pulse frequency: $50 \mathrm{kHz}$, velocity: $200 \mathrm{~mm} \cdot \mathrm{s}^{-1}$, number of repetitions: 1) to produce micrometer-scaled grooves. A characterization of the surface was carried out by laser scanning microscopy for the determination of 3D information (see Fig. 1a) and surface profiles. The surface structure is quite uniform across the surface. Due to the limitations of laser scanning microscopes, microsections were also used to detect the undercuts of the surface structures that form at the exit edge towards the surface (see Fig. 1b).
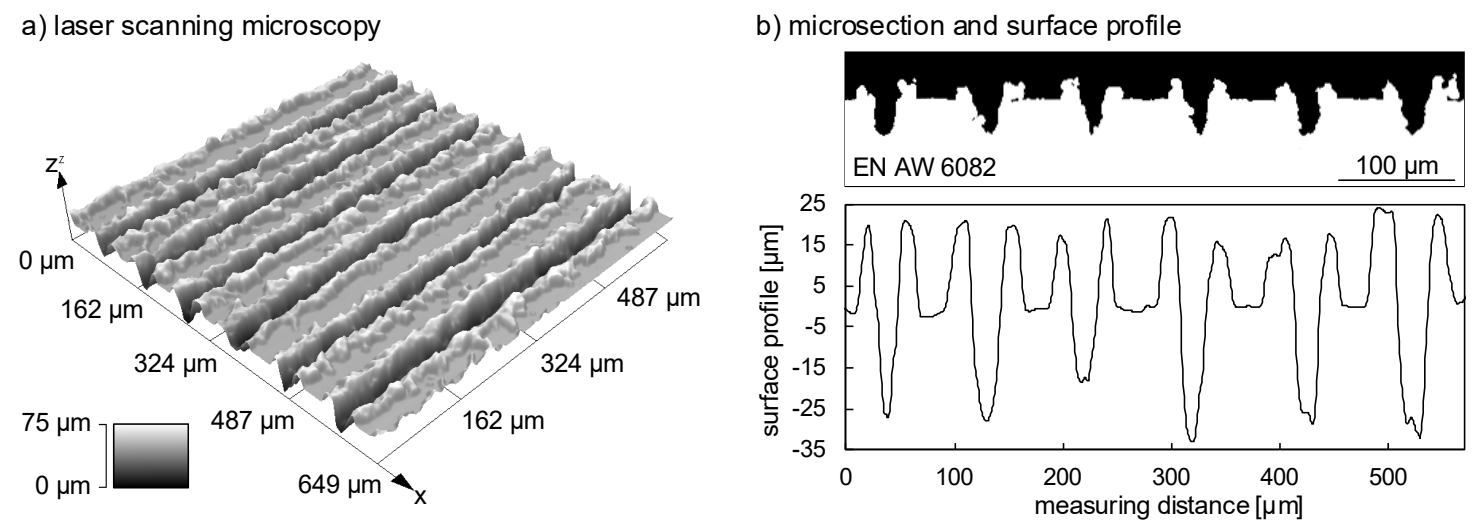

Fig. 1. a) Laser scanning microscopy of the metal surface, b) microsection and surface profile

\section{Spot joints}

The investigations were carried out on idealized spot joints. Besides the use of a semi-infinite body in case of thermoplastic materials, an overlap area of $100 \times 75 \mathrm{~mm}^{2}$ was applied to ensure a mostly uniform heat conduction. A clamping device ensures a reproducible positioning of the joining partners and allows the measurement of the applied clamping force due to integrated load cells (recording rate: $100 \mathrm{~Hz}$ ). The joining process was realized by a diode laser (Laserline LDM 3000, average wavelength: $980 \mathrm{~nm}$, beam power: $1 \mathrm{~kW}$, focal diameter: $5.3 \mathrm{~mm}$ ). Joining times $\left(\mathrm{t}_{\mathrm{L}}\right)$ were realized from $1 \mathrm{~s}$ up to $10 \mathrm{~s}$. The joint configuration is shown in Fig. 2. For mechanical testing, the specimens were cut to size after the joining process and a tensile-shear-test was carried out using a universal testing machine (Hegewald \& Peschke Inspekt $145520 \mathrm{kN}$, free clamping length: $30 \mathrm{~mm}$, test speed: $\left.10 \mathrm{~mm} \cdot \mathrm{min}^{-1}\right)$.
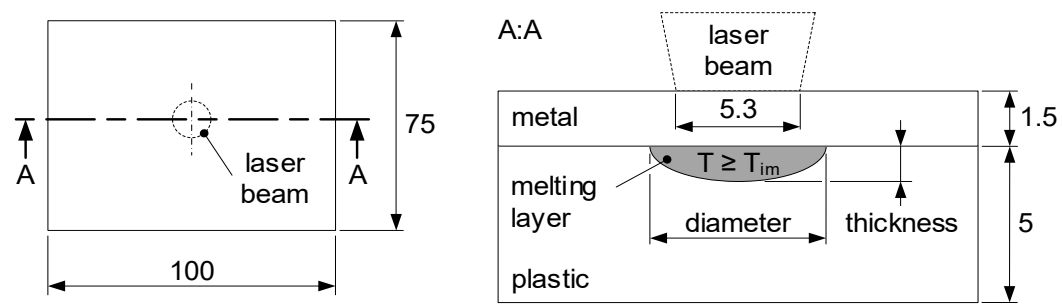

Fig. 2. Joint configuration for spot joints (schematic view, not to scale) 


\section{Half-section setup}

For further investigations on spot joints, a half-section setup was used which allows high-speed recordings (Photron SA-X2, recording rate: 1,000 fps) directly in the joining zone. A schematic view on the half-section setup is given in Fig. 3. Further information regarding the validation of the setup are published in [13]. The images were rotated $180^{\circ}$ for a better understanding compared to the arrangement of both joining partners during the recording and adjusted in contrast/brightness.

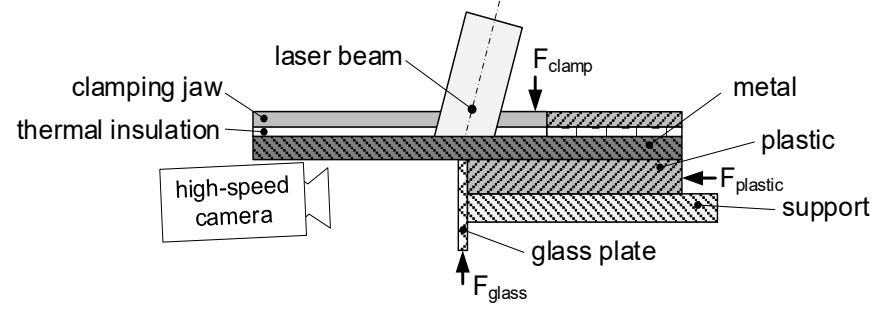

Fig. 3. Schematic view of the half-section setup for thermal joining (schematic view, not to scale)

\section{Thermal Model and Numerical Simulation}

A thermal model was used in order to investigate the temperature distribution within the joining zone based on the isotherms $\mathrm{T}_{\mathrm{im}}, \mathrm{T}_{\mathrm{em}}$ and $\mathrm{T}_{\mathrm{d}}$ determined by thermal analysis. The geometry, the physics as well as the initial and boundary conditions are described in more detail in [14]. The simulations were carried out in Comsol Multiphysics 5.2a.

\section{Results and Discussion}

\section{Joining Zone in Thermal Joining}

The joining zone between thermally joined metal-plastic components can be described as model concept based on the investigations on the temperature distribution and morphology in [14-16]. The absorption of laser radiation on the upper side of the metal creates a transient temperature field in metal and plastic. Starting from the metal surface, as location of the highest temperatures in heat conduction joining, the temperature field describes the time- and location-dependent formation of the melting layer. On the one hand, it is necessary to exceed certain temperatures for joining in order to allow the surface structures to be wetted and filled by the plastic. On the other hand, the temperatures may lead to decomposition of the plastic. Due to the possible application of hybrid composites in visible areas, melting of the metal is not considered in detail at this point.

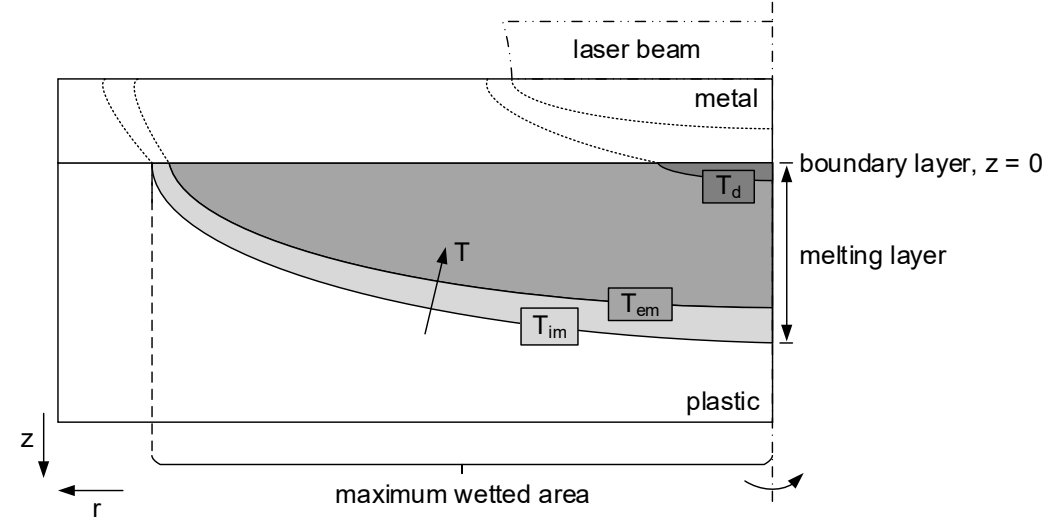

Fig. 4. Model of a rotationally symmetrical melting layer based on characteristic isotherms

From this, different areas within the joining zone can be derived, which are described by the temperature field. The temperatures $T_{i m}, T_{e m}$ and $T_{d}$, determined in thermal analyses, are set to the joining zone as characteristic isotherms. Fig. 4 schematically illustrates this model approach using a rotationally symmetrical joining zone for spot joints with the corresponding isotherms in the thermoplastic material. The beginning and the end of the melting interval are highly important for the joining zone, as a precise statement of the material behavior is possible in contrast to the sole 
consideration of the peak temperature $\mathrm{T}_{\mathrm{pm}}$. On the one hand, for temperatures higher than $\mathrm{T}_{\mathrm{em}}$, the material is completely molten. On the other hand, the plastic is only partially molten between $\mathrm{T}_{\mathrm{em}}$ and $\mathrm{T}_{\mathrm{im}}$, but even in this temperature interval, wetting or structural filling of the metal surface may occur (depicted as maximum wetted area). Therefore, the entire area above $T_{i m}$ is considered within the melting layer. Further investigations on the morphology and the heat-affected zones in plastic and metal are not the subject of the investigations at this point and are discussed in detail in [15].

Based on the numerical simulation, the partially molten area as well as the completely molten area can be quantified (the validation of the model was published in [14]). Investigations on non-polar PP were carried out to exclude any effect of physico-chemical interactions between the molten thermoplastic and the metal surface. Thereby, the melting interval of PP occurs at relatively low temperatures $\left(\mathrm{T}_{\mathrm{im}}=126{ }^{\circ} \mathrm{C}, \mathrm{T}_{\mathrm{em}}=178{ }^{\circ} \mathrm{C}\right)$ and at the same time is relatively wide in the range $\mathrm{T}_{\mathrm{im}}$ to $\mathrm{T}_{\mathrm{em}}$ with approximately $52 \mathrm{~K}$. Fig. 5 depicts the melting layer for a joining time of $5 \mathrm{~s}$.

The size of the entire melting layer is very limited and shows a thickness of approx. $520 \mu \mathrm{m}$. The diameter of the melting layer is significantly larger than the thickness of the melting layer due to the higher thermal conductivity of the metal sheet compared to the thermoplastic. The numerical simulation revealed that only approx. $56 \%$ of the melting layer thickness and about $59 \%$ of the melting layer diameter contained completely molten material $\left(\mathrm{T} \geq \mathrm{T}_{\mathrm{em}}\right)$. Thereby, bubble formation, which could counteract the structure filling [13], is avoided due to joining temperatures below the degradation temperature of PP $\left(390^{\circ} \mathrm{C}\right)$. In the experiment, a shift from complete to incomplete structure filling occurs in the transition zone between $T_{e m}$ and $T_{i m}$ (Fig. 5), although a sufficient amount of molten material is required. This behavior is considered in the chapter after next.

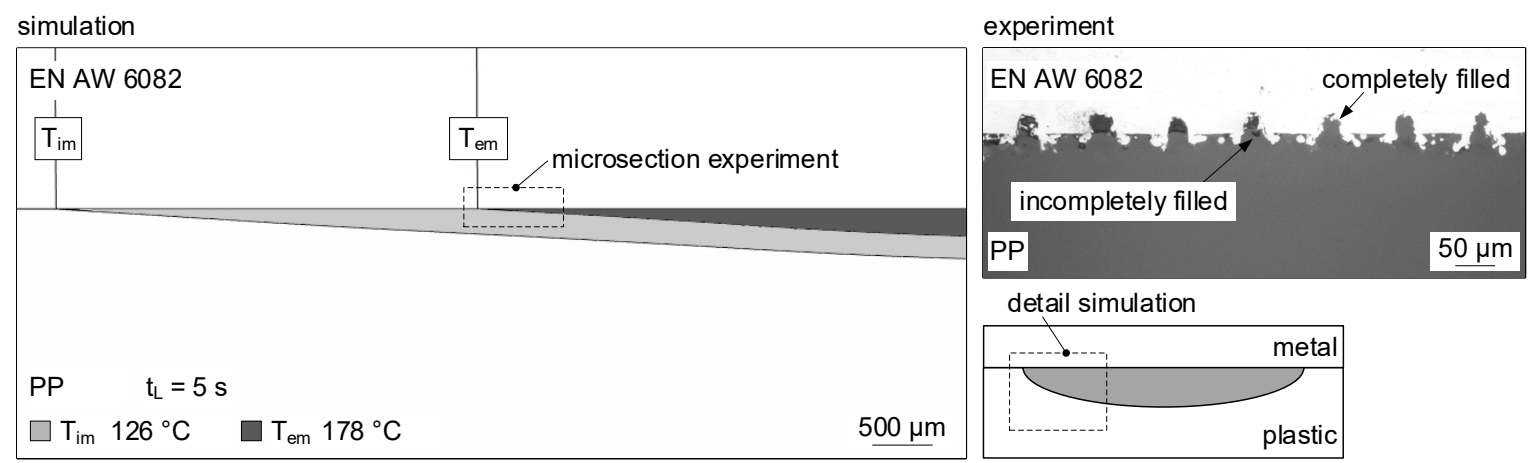

Fig. 5. Edge of melting layer in simulation and microsection from the experiment $\left(t_{L}=5 \mathrm{~s}\right)$

It should be noted that the filling of the surface structures is influenced by simultaneously occurring effects like increasing volume, joining pressure and viscosity. For a better understanding of this effects, the thermal expansion as well as the temperature distribution are investigated separately.

\section{Effect of Thermal Expansion}

The specific volume of the thermoplastic material PP as a function of the temperature is given in Fig. 6. For an increased understanding of the joining process, the graph is standardized to the starting point of the melting interval $\left(\mathrm{T}_{\mathrm{im}}\right)$. For temperatures below $\mathrm{T}_{\mathrm{im}}$, the specific volume is increasing linearly due to thermal expansion. Especially during the phase transition solid-liquid starting from $T_{\mathrm{im}}$ - the specific volume is increasing significantly. By reaching $\mathrm{T}_{\mathrm{em}}$, approx. $52 \mathrm{~K}$ above $\mathrm{T}_{\mathrm{im}}$, a range of linearly increasing specific volume of the completely molten material follows.

This effect has a decisive influence on the joining process and the filling of the surface structures. Based on the half-section setup, high-speed recordings were carried out directly in the joining zone. The volume increase leads to several effects. Fig. $6 \mathrm{~b}$ shows the edge of the melting layer during the interval between switch-off of the laser and the technically last possible recording time after $21 \mathrm{~s}$. At the time $t=10 \mathrm{~s}$, the melting layer is clearly identifiable to the solid phase of PP and aluminum. Despite the complete overlap, both materials are not in full contact. This effect is based on the described volume increase in the phase transition, resulting in a gap between plastic and metal which is only bridged by molten material. The heat conduction is therefore also limited to this area. 

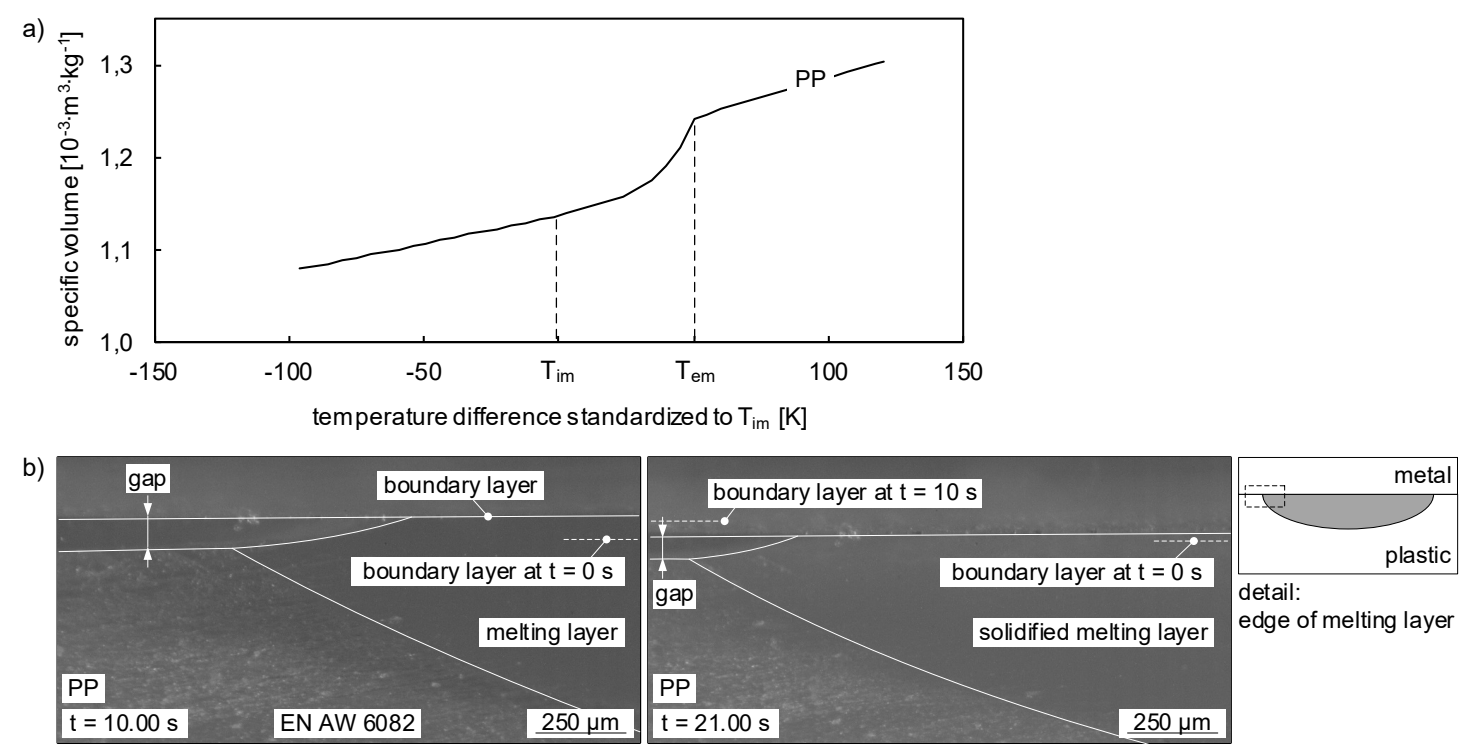

Fig. 6. a) Specific volume of PP (see [17]) b) high-speed recordings in half-section setup $\left(t_{L}=10 \mathrm{~s}\right)$

In addition to the formation of the gap, a displacement of the boundary layer during the process can be observed. During the melting process, the boundary surface moves in the direction of the aluminum, with the resulting force acting against the clamping device (Fig. 7a). A maximum position change of approximately $53 \mu \mathrm{m}$ is reached at the end of the laser process $(\mathrm{t}=10 \mathrm{~s})$. During the cooling phase respectively solidification, the interface approaches the initial position again $(\mathrm{t}=21 \mathrm{~s})$. However, a shift of approximately $10 \mu \mathrm{m}$ remains. The effects of bubble formation, variations in crystal modification and changes in the degree of crystallization could be excluded as mechanisms of the permanent volume increase by referencing studies on polyamide 6 and polyamide 6.6 [15]. A possible explanation is the orientation of macromolecules in the surface layer, which is introduced in the manufacturing process of the plastic sheets [18]. This orientation can be removed during the melting process, resulting in a swelling of the plastic and an increased specific volume compared to the adjacent base material.

The increase in volume can also be tracked in the joining process for spot joints using integrated load cells in the clamping device. Fig. $7 \mathrm{~b}$ shows the increase in force $\Delta \mathrm{F}$ compared to the start of the joining process with the clamping force $\mathrm{F}_{\text {clamp. }}$. At the beginning of the joining process, a significant rise in force occurs, due to the thermal expansion of the material and the solid-liquid phase transition in particular. At the end of the joining process, an increase of approximately $35 \mathrm{~N}$ up to $40 \mathrm{~N}$ compared to the initial level can be determined. Due to heat conduction, the temperature field propagates in the direction of the clamping elements with a delay, which explains the increase in force after the end of the joining process. At the maximum, approximately $70 \mathrm{~N}$ are reached. During cooling, the force decreases against a limiting value without dropping to the initial level. In the example shown, an additional load of approximately $38 \mathrm{~N}$ remains because the boundary surface is permanently displaced. The effect is detectable for all investigated materials but depends on a corresponding melt volume and the temperature distribution. It should be noted that a single spot joint is responsible for such a large effect. For increasing melt volumes, e. g. for line joints in overlap configuration, an even greater effect can therefore be expected.

It can be assumed that the joining pressure must be applied at the time of process start in order to ensure heat transfer between the two materials, while the increase in volume due to thermal expansion or phase transition significantly supports the structural filling of the metal surface. Based on this phenomena, different influences arise on the joining process. Firstly, the heat transfer between both joining partners in the area of the formed melt bridge is positively influenced. Secondly, the increase in volume of the melt can enable better filling of the surface structures, since the melt itself builds up the pressure. 
In addition to thermal expansion, the temperature distribution within the joining zone plays an important role in the penetration of surface structures. For this reason, further investigations on the influence of the temperature distribution and the joining time were carried out.
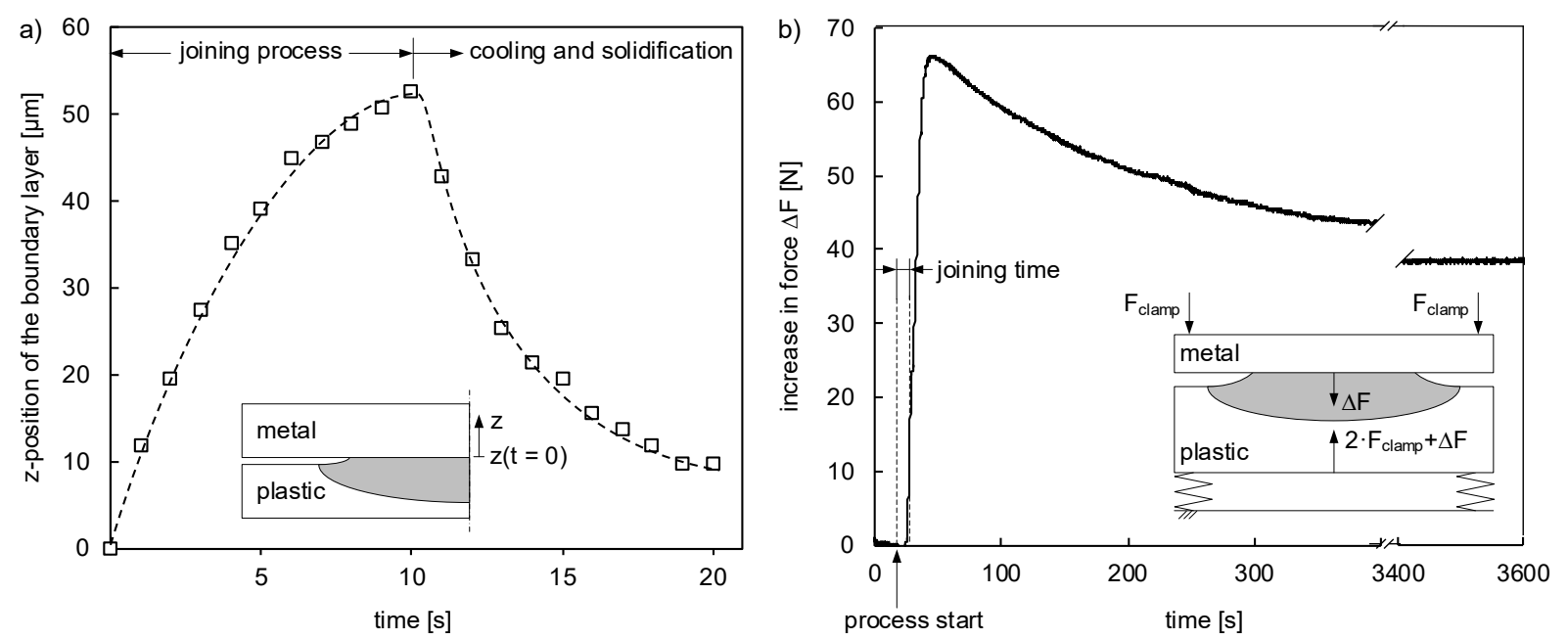

Fig. 7. a) Movement of the boundary layer in half-section setup b) increase in force $\Delta F\left(t_{L}=10 \mathrm{~s}\right)$

\section{Effect of Joining Times and Temperature Distribution}

A separate consideration of temperature distribution and structure filling was carried out by further experiments. In order to determine the structure penetration and the resulting tensile shear strength due to the form fit, the structured surface area must always be completely within the melting area for all investigated joining times. This allows the complete filling of the structures by molten plastic. With joining times of $t_{L}=1 \mathrm{~s}$, the melt zone of PP reaches a diameter of approximately $9 \mathrm{~mm}$ at the boundary layer between metal and plastic. Therefore, the described approach is implemented by defining a structured area with a diameter of $7.5 \mathrm{~mm}$. At the same time, any positioning inaccuracies or process deviations were taken into account by providing a tolerance of $\pm 0.75 \mathrm{~mm}$ in relation to the expected melt zone diameter. Fig. 8 shows the shear tensile strength as a function of the joining time $t_{L}$. In addition, the fractions of completely molten plastic $\left(T \geq T_{e m}\right)$ within the structured area at the interface are compared with the achieved shear tensile strengths. Characteristic fracture surfaces are assigned to the results after the mechanical testing. $\mathrm{A}_{\mathrm{s}}$ denotes the area of the structured surface of the metal $(\varnothing 7.5 \mathrm{~mm}), A_{p}$ the fracture surface with penetrated surface structures and $A_{d}$ the area with bubble formation due to thermal decomposition.

For joining times of $1 \mathrm{~s}$, a shear tensile strength of approximately $21 \mathrm{~N} \cdot \mathrm{mm}^{-2}$ is achieved. The area share of completely molten plastic could be determined by numerical simulation and is about $45 \%$, while the fracture surface indicates a proportion of about $80 \%$ filled surface structures $\left(A_{p}\right.$ in fracture behavior a). The surface structure is thus also filled proportionally with partially molten material. With increasing joining time $(2 \mathrm{~s}, 5 \mathrm{~s})$, the tensile shear strength reaches a plateau of approximately $30 \mathrm{~N} \cdot \mathrm{mm}^{-2}$ when the area share of completely molten material reaches $100 \%$. This ensures a complete structural filling and the fracture surface of the penetrated surface corresponds to the entire structured surface (fracture behavior $b$ ). This clarifies that a maximum form fit can be achieved even for short joining times with complete melting of the material. This also indicates that longer joining and wetting times are not required for form-fit composite production. As the joining times increase to $10 \mathrm{~s}$, the achievable tensile shear strength drops to approximately $25 \mathrm{~N} \cdot \mathrm{mm}^{-2}$. Again, the thermoplastic is completely molten in the area of the surface-structured interface, but bubbles form within the melting zone, which reduces the bonding cross-section between both joining partners. Since PP does not dissolve water, thermal degradation can be assumed to be the cause [13]. In the numerical simulation, peak temperatures occur in the center of the melting layer that are above the decomposition temperature $\mathrm{T}_{\mathrm{d}}$. 
This illustrates that a structure filling can be achieved even for short joining times and independent of the interactions between polymer melt and metal. A complete penetration of the metal surface with thermoplastic material is reliably achieved by exceeding the end of the melting interval $\mathrm{T}_{\mathrm{em}}$. The influence of interaction effects, the melting interval width or the viscosity is subordinate at this point. A complete structure filling to maximize the form fit can therefore be achieved by temperature control and does not require long joining times.
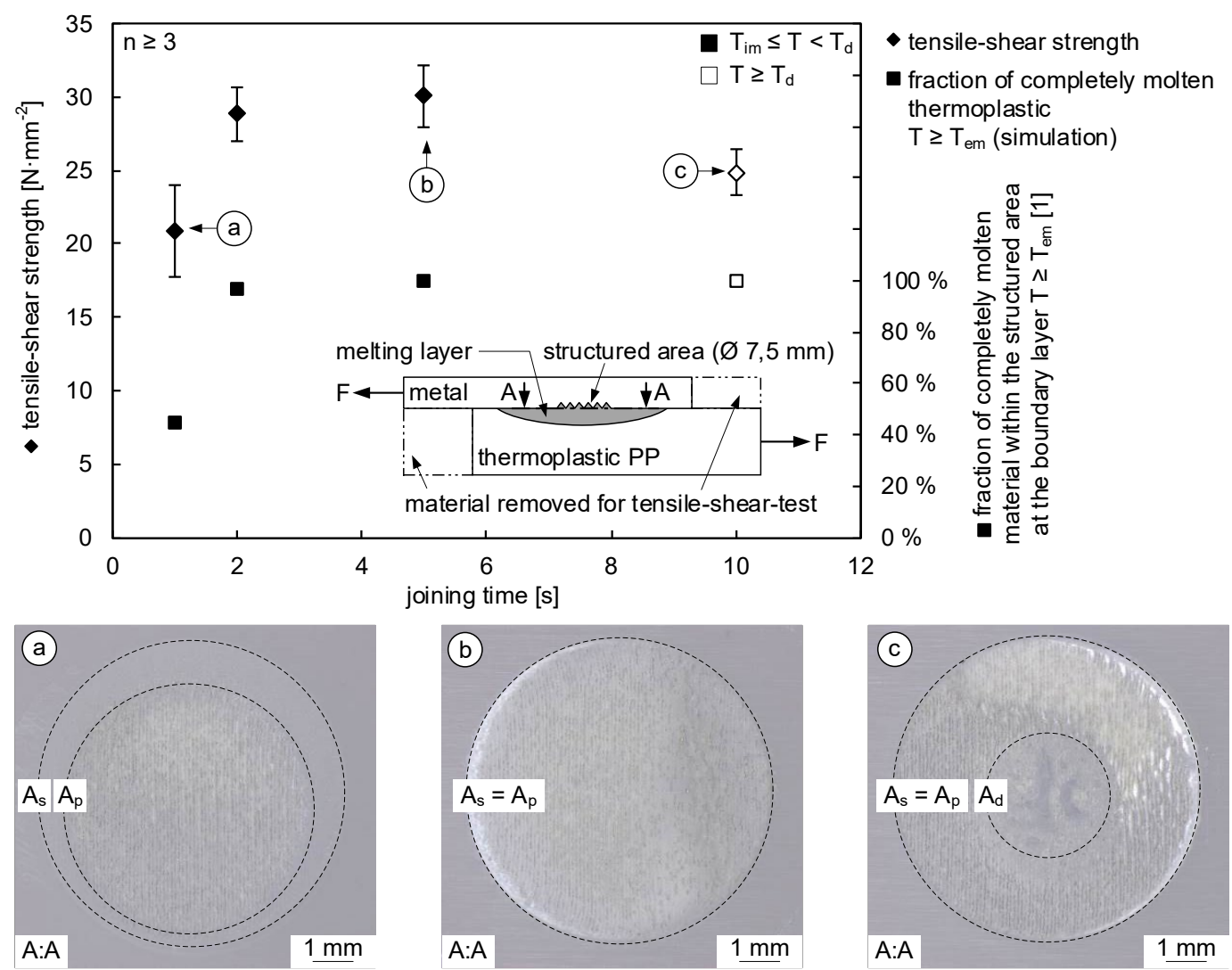

Fig. 8. Dependency of tensile-shear strength on joining time and penetrated surface area

\section{Summary}

In this paper, investigations regarding the time- and temperature-dependent penetration of surface structures in thermal joining were carried out. The penetration of surface structures was identified as main parameter for achieving a high strength based on the form fit between metal and plastic.

Starting with a model concept of the joining zone, different areas are identified and verified by numerical simulation. The melting layer consists of different areas depending on the temperature distribution. Thereby, the phase transition solid-liquid was identified as significant parameter for surface penetration due to the increase in specific volume and its effect on joining pressure which supports surface penetration. The effects were traced by use of a half-section setup.

The interaction between temperature and time on surface penetration was carried out by experimental investigations and accompanying studies based on numerical simulation. Thereby, the temperature distribution was identified as main parameter, because a sufficiently high temperature above $T_{e m}$ ensures short joining times. In contrast to the state of the art, the joining time in the considered interval $(1 \ldots 10 \mathrm{~s})$ could be excluded as influencing factor. It should be noted that supporting mechanisms, e. g. adhesion forces between polymer melt and the metal surface, can be excluded due to the chosen thermoplastic material.

Further investigations will be carried out with various thermoplastic materials in order to understand and generalize the influence of material properties, e. g. viscosity and the effect of different structure geometries. 


\section{References}

[1] S. Katayama, Y. Kawahito, Y. Niwa, S. Kubota, Laser-Assisted Metal and Plastic Joining, Proceedings of the LANE 2007: Laser Assisted Net Shape Engineering 5 (2007) 41-51.

[2] M. Stambke, K. Schricker, J. P. Bergmann, A. Weiß, Laser-based joining of metal-thermoplastic tailored welded blanks, Welding in the World 61 (2017), 563-573.

[3] G. Habenicht, Kleben, Springer Berlin Heidelberg (2009)

[4] A. Heckert, M. F. Zaeh, Laser Surface Pre-treatment of Aluminium for Hybrid Joints with Glass Fibre Reinforced Thermoplastics, Physics Procedia 56 (2014) 1171-1181.

[5] S. M. Goushegir, Friction Spot Joining of Metal-Composite Hybrid Structures, HelmholtzZentrum Geesthacht, HZG REPORT 2015-5 (2015)

[6] A. Heckert, C. Singer, M. F. Zaeh, R. Daub, T. Zeilinger, Gas-tight thermally joined metalthermoplastic connections by pulsed laser surface pre-treatment, Physics Procedia 83 (2016) 10831093.

[7] K. Schricker, M. Stambke, J. P. Bergmann, Laser-Based Joining of Thermoplastics to Metals: Influence of Varied Ambient Conditions on Joint Performance and Microstructure, International Journal of Polymer Science (2016)

[8] M. Bielenin, K. Szallies, J. P. Bergmann, C. Neudel, Single Side Resistance Spot Welding of Polymer-Metal-Hybrid Structures, Proceedings Euro Hybrid Materials \& Structures (2016) 236-240.

[9] C. Ageorges, L. Ye, Resistance Welding of Metal/Thermoplastic Composite Joints, Journal of Thermoplastic Composite Materials 14 (2001) 449-475.

[10]DIN EN ISO 11357-1, Plastics - Differential scanning calorimetry (DSC). Part 1: General principles (2010)

[11] G. W. Ehrenstein, G. Riedel, P. Trawiel, Thermal Analysis of Plastics, Hanser Munich (2004)

[12]DIN 51006, Thermal analysis (TA) - Thermogravimetry (TG) - Principles (2005)

[13]K. Schricker, S. Diller, J. P. Bergmann, Bubble formation in thermal joining of plastics with metals, Procedia CIRP 74 (2018) 518-523.

[14]K. Schricker, J. P. Bergmann, Determination of sensitivity and thermal efficiency in laser assisted metal-plastic joining by numerical simulation, Procedia CIRP 74 (2018) 511-517.

[15]K. Schricker, J. P. Bergmann, M. Hopfeld, L. Spieß, Characterization of the joining zone in laser direct joining between thermoplastics and metals, Proceedings Hybrid Materials \& Structures (2018) 210-215.

[16]K. Schricker, M. Stambke, J. P. Bergmann, Experimental investigations and modeling of the melting layer in polymer-metal hybrid structures, Welding in the World 59 (2015) 407-412.

[17] Autodesk, Autodesk Moldflow material library, 2017

[18]B. A. G. Schrauwen, L. C. A. v. Breemen, A. B. Spoelstra, L. E. Govaert, G. W. M. Peters, H. E. H. Meijer, Structure, Deformation, and Failure of Flow-Oriented Semicrystalline Polymers, Macromolecules 37 (2004) 8618-8633. 